\title{
DREAM OR REALITY: COMPLETE BASIS SET FULL CONFIGURATION INTERACTION POTENTIAL ENERGY HYPERSURFACES
}

Attila G. Császár, György Tarczay

Department of Theoretical Chemistry, Eötvös University

P. O. Box 32, H-1518 Budapest 112, Hungary

Matthew L. Leininger

Sandia National Laboratory, MS 9217, Livermore, CA 94551-0969

Oleg L. Polyansky, Jonathan Tennyson

Department of Physics and Astronomy, University College London

Gower St., London WC1E 6BT, U.K.

Wesley D. Allen

Center for Computational Quantum Chemistry, Department of Chemistry,

University of Georgia, Athens, GA 30602, U.S.A.

\section{OVERVIEW}

All knowledge about molecules outside of our solar system comes from study of their spectra. Although laboratory measurements are usually the prime source for the data needed to interpret astronomical observations, there are a number of reasons why theory has played, and will continue to play, a central role in the study of molecules in space.

Astronomical environments, such as those found in the interstellar medium, are very different from those on earth. This dissimilarity leads to fundamentally different chemistry and to the production of species that can be hard to create in the laboratory. Theory can play an impor- 
tant role in both predicting main features of spectra of such species or in looking for possible spectral matches.

Even where laboratory spectra have been recorded for a particular species, this data may only be partial. One such situation, which is particularly common for unstable or reactive species, is that wavelengths can be measured to high accuracy but there is no information on transition probabilities or line strengths. Line strength data are essential for obtaining useful information from astronomical spectra. Without them observation of a spectrum simply indicates that a particular species is present. Use of the line strengths gives column densities as well as the local temperature if thermodynamic equilibrium prevails, or other environmental information if a non-equilibrium environment is being probed. The $\mathrm{H}_{3}^{+}$molecule provides a good example of this situation. The laboratory infrared spectrum of $\mathrm{H}_{3}^{+}$is well known. Its original measurement [1], and indeed subsequent experiments, relied heavily on $a b$ initio theory. $\mathrm{H}_{3}^{+}$has now been observed in giant planets, diffuse and dense clouds in the interstellar medium [2], and the remnants of a supernova [3]. The infrared spectrum of $\mathrm{H}_{3}^{+}$has provided a particularly powerful handle on ionospheric activity in giant planets [4], giving detailed information on a whole variety of effects including, for example, the electrojets which power the auroral activity on Jupiter. Yet while the characteristic wavelengths used for observations of $\mathrm{H}_{3}^{+}$all come from experiment, all line strengths used to model the spectra are theoretical and are the result of $a b$ initio calculations [5].

Some astronomical applications, stellar models being a classic example, are particularly demanding on spectroscopic data. The stellar opacity problem for stars hotter than our own Sun was only solved by the systematic calculation of large quantities of spectral data for atoms and atomic ions by the international Opacity Project Team [6]. The spectroscopic properties of cooler stars are dominated by molecular absorptions [7]. However, to model the role of a triatomic molecule such as $\mathrm{H}_{2} \mathrm{O}$ or $\mathrm{HCN}$, which are important components of oxygen- and carbon-rich cool stars respectively, it may be necessary to consider up to a billion vibration-rotation transitions. The laboratory measurement and analysis of a dataset of transitions of this size is completely impractical. Recently this task has been addressed by theory, where large linelists of rotation-vibration transitions have been produced for $\mathrm{HCN}[8]$ and $\mathrm{H}_{2} \mathrm{O}$ $[9,10]$. It has been found that including these transitions can fundamentally alter conclusions about the structure of the stellar atmosphere [8]. Despite the use of very high level theory and large datasets, recent stellar models have shown, for example, that the best water linelists are still not adequate for reproducing the observed spectra [11]. Large datasets of 
energy levels produced by theory can also be used to model other astronomically important properties such as temperature-dependent partition functions [12] or equilibrium constants [13].

The vibration-rotation spectrum of water is perhaps the most important and intensely studied of all molecular spectra. For instance, water vapour is thought to be responsible for absorbing $70 \%$ of the sunlight lost in a cloudless atmosphere [14]. Many decades of work have focused on measuring, analysing, and modeling the spectrum of water. In something of a theoretical triumph, Polyansky et al. $[15,16]$ have used the combination of high level $a b$ initio electronic structure and variational nuclear motion calculations to assign 1687 transitions to water in the sunspot spectra recorded in the $10-20 \mu \mathrm{m}$ region. Yet these transitions represent only about $15 \%$ of the clearly resolved features observed in sunspots in this spectral region. It is likely that nearly all the unassigned features are also due to water. Further significant progress in assigning these features will require corresponding theoretical developments. It is therefore important to consider all possible factors which influence the $a b$ initio calculation of transition frequencies. The major factor determining the accuracy of a computed rotation-vibration spectrum is the potential energy hypersurface (PES) employed. State-of-the-art $a b$ initio electronic structure calculations have become able to predict vibrational band origins (VBOs) of triatomics, like water, to within a few wavenumbers, and other spectroscopic properties with similar $(\sim 0.1 \%)$ accuracy $[10,15-21]$. Standard treatments of molecular electronic structure theory tacitly neglect several physically significant factors which become important in high-accuracy theoretical work: core-valence electron correlation, coupling between electronic and nuclear motion, part of which is considered in the so-called diagonal Born-Oppenheimer correction (DBOC), and relativistic corrections. A number of groups have recently started exploring the validity of the Born-Oppenheimer approximation when calculating vibration-rotation spectra, using water as the test molecule [10, 22]. Electronic relativistic effects [23] are also receiving considerable interest, although attention is only starting to be paid to the possible spectroscopic consequences of the relativistic correction for light molecules [24-30].

\section{INTRODUCTION}

Ever since the Egyptians invented them, maps (pinax for Greeks, orbis pictus or tabula for Romans) have helped mankind to find directions in the known world and, at the same time, to make explorations 
of the unknown world. In fact, the history of maps is intertwined with the history of mankind.

The concept of potential energy hypersurfaces $[31,32]$ is as fundamental to the understanding of most modern branches of chemistry and physics including almost the whole of spectroscopy and kinetics, as maps are for our everyday life. Nevertheless, PESs exist only within the socalled Born-Oppenheimer (BO) separation of electronic and nuclear motion [33, 34]. Following this separation, potential energy hypersurfaces for nuclear motion arise describing the variation in the total electronic energy of a chemical system as a function of its geometry (internal coordinates of the $N$ constituent nuclei). Usually, attention is focused on cases where a single BO-PES is sufficiently uncoupled from other surfaces (electronic states) that their interaction may be ignored.

Despite their great importance, production of detailed and accurate maps took quite a long time to achieve. In a similar manner, due to methodological problems and insufficient computational power, ab initio quantum mechanical study of PESs remained unrealistic until about 1970.

Recent developments in electronic structure theory and computer technology have facilitated the computations necessary for the theoretical determination of high accuracy potential energy hypersurfaces. Theory is now capable, over a rather large range of geometries, of obtaining near chemical accuracy $( \pm 1 \mathrm{kcal} / \mathrm{mol})$ for the relative energies of small and medium-sized molecular systems. In favorable cases this translates into an accuracy of a few $\mathrm{cm}^{-1}$ for rovibrational eigenstates computed from these PESs, as shown below.

The computational errors in today's approximate solutions to the time-independent non-relativistic electronic Schrödinger equation result from the truncation of the atomic orbital one-electron basis and the truncation of the $n$-electron basis of all Slater determinants that constitute the full configuration interaction (FCI) wave function. Techniques which aim to achieve chemical accuracy for energetic quantities include the Gaussian- $n$ [35-38], the complete basis set (CBS) [39, 40], and the $\mathrm{W}-n$ [41] model chemistries. These approaches may rely on empirical parameters which are optimized to minimize the errors for a given test set of molecules. An alternative parameter-free method is the focalpoint $[42,43]$ approach (see Section 4 below), which seeks to achieve the complete one-electron basis set (CBS) and $n$-electron (FCI) limit by performing a series of electronic structure computations employing convergent basis sets and correlation methods. The previously mentioned model chemistries may be considered as approximations to the focalpoint approach, and are therefore more computationally efficient but 
may be less accurate for certain troublesome cases. Furthermore, the focal-point approach allows efficient estimation of the remaining computational error [43-45] for the given problem, while in the case of model chemistries one needs to rely on average error estimates.

The determination of the complete one-electron basis set limit has received considerable attention in recent years [41,43-51]. Formulas have been advanced providing an estimate of the CBS energy limit from calculations employing systematically constructed families of basis sets (e.g., the correlation-consistent (cc) basis sets of Dunning and co-workers [52-54]. These studies show that different levels of electronic structure theory follow distinct basis set extrapolation patterns; most notably, Hartree-Fock (HF) energies converge almost exponentially toward the CBS limit [49], while correlation energies seem to follow an $X^{-3}$ dependence [43, 46], where $X$ is the cardinal number of the cc basis sets [52]. Therefore, the expressions $E^{X}=E_{\mathrm{CBS}}+a \exp (-b X)$ and $E^{X}=E_{\mathrm{CBS}}$ $+c X^{-3}$, where $E_{\mathrm{CBS}}$ is the extrapolated energy, $E^{X}$ denotes energies obtained from correlation-consistent basis sets with cardinal number $X$, while $a, b$, and $c$ are fitting constants, are now commonly employed for estimating the complete basis set $\left(E_{\mathrm{CBS}}\right)$ Hartree-Fock and correlation energies, respectively. Accordingly, the above formulas are employed throughout this study for CBS extrapolations. Note that certain correlation contributions may behave differently, such as the relativistic 2-electron Darwin correction [50], which scales as slowly as $X^{-1}$.

Of equal importance is the determination of the FCI limit in a given one-particle basis from approximate $n$-electron methods. Numerous studies have shown the diminishing energy contribution of higher excitation levels. Coupled-cluster (CC) methods [55] including triple excitations [56-58] or configuration interaction with up to quadruple substitutions [59] typically provide accurate and reliable approximations to the FCI energy, in contrast to Møller-Plesset perturbation methods [60]. In particular, the CCSD(T) [56] approach represents a good compromise between cost and accuracy for predicting high-quality energies and properties [61]. Nevertheless, to compute energies to better than chemical accuracy it is necessary to consider the energy contributions from higher-order excitations. For the estimation of higher-order correlation effects neglected in the $\operatorname{CCSD}(\mathrm{T})$ and CCSDT approaches, we present here a technique called scaled higher-order correlation energy (SHOC) ([62]; see Section 5). It is important to note that if SHOC is carried out at the extrapolated CBS limit, the penultimate (CBS FCI) solution of the non-relativistic electronic Schrödinger equation may be closely approximated. 
Reproduction of spectroscopic observations provides the most stringent test for checking the intrinsic accuracy of any $a b$ initio PES. Therefore, in this paper we primarily focus on the ab initio generation of PESs and on the accuracy of the subsequent prediction of the rovibrational spectra of two molecules of recent interest for us and to astronomers: $\mathrm{H}_{2} \mathrm{O}$ and $\mathrm{H}_{2} \mathrm{~S}$.

\section{COMPUTATIONAL DETAILS}

Detailed documentation of the computational methods described in the text can be found in the original papers [27-29, 43-45]. When excerpts from previous work are presented, the notation utilized in the source is not modified. A brief summary of computational details and programs is provided to aid readers already familiar with most of the notation.

\subsection{ELECTRONIC STRUCTURE CALCULATIONS}

The configuration interaction (CI) calculations, including full CI (FCI), utilized the DETCI code [59] interfaced with the PSI3 program system [63], while the ACESII code [64] has been employed for the coupledcluster (CC) calculations. Kinetic relativistic effects have been gauged by first-order perturbation theory applied to the one-electron massvelocity and the one- and two-electron Darwin terms [23], as implemented via the recipe of Klopper [65] within the DALTON program system [66]. Calculation of the Gaunt correction surface utilized the program package MOLFDIR [67, 68].

The correlation-consistent (cc-pVXZ) basis sets of Dunning and coworkers [52-54], as well as their augmented (aug-cc-pVXZ), core-valence (cc-pCVXZ), and uncontracted variants, have been employed almost exclusively in our studies.

\subsection{NUCLEAR MOTION CALCULATIONS}

The rovibrational energy states have been determined using an exact kinetic energy (EKE) operator expressed in Radau coordinates augmented with the fitted $a b$ initio PESs. The nuclear motion calculations utilized the DVR3D program suite [69] and optimized basis sets [70, 71]. Rovibrational calculations are only reported here for the $\mathrm{H}_{2}^{16} \mathrm{O}$ and $\mathrm{H}_{2}^{32} \mathrm{~S}$ isotopomers. All calculations presented used a hydrogen mass midway between the atomic and nuclear value, as recommended by Zobov et al. $[22]$. 


\section{THE FOCAL-POINT APPROACH (FPA)}

Characteristics of the focal-point approach [42, 43], comprising the dual extrapolation to the one- and $n$-particle $a b$ initio limits, are as follows: (a) use of a family of basis sets which systematically approaches completeness (e.g., the cc-pVXZ, aug-cc-pVXZ, and cc-pCV $X Z$ zets of Dunning and co-workers [52-54]; (b) application of low levels of electronic structure theory with basis sets as large as possible (typically direct Hartree-Fock (HF) and MP2 computations with hundreds of basis functions); (c) higher-order valence correlation treatments [CCSD $(\mathrm{T})$, CCSDT, BD(TQ), MP4, MP5, and preferably FCI] with the largest possible basis sets; (d) construction of a two-dimensional extrapolation grid based on the assumed additivity of correlation increments to energy differences; and (e) avoidance of empirical corrections. Focal-point investigations [42-45, 72-74] and numerous other theoretical studies have shown that even in systems without particularly heavy elements, account may also be needed for core correlation and relativistic phenomena, as well as the diagonal Born-Oppenheimer correction (DBOC). Auxiliary shifts for these effects are appended to valence-only focal-point analyses not only to ensure the highest possible accuracy but also to enhance our understanding of the manifestation of these effects for different chemical applications (e.g., conformational energy and dissociation energy calculations).

The focal-point scheme assumes that the correlation energy increments have rather different convergence characteristics, with the higherorder correlation increments showing diminishing basis set dependence. This assumption has allowed efficacious estimation of molecular barriers at the CBS FCI limit for $\mathrm{H}_{2} \mathrm{O}, \mathrm{H}_{2} \mathrm{~S}, \mathrm{NH}_{3}, \mathrm{SiH}_{3}{ }^{-}, \mathrm{C}_{2} \mathrm{H}_{6}, \mathrm{HCOOH}$, $\mathrm{HNCO}$, and $\mathrm{SiC}_{2}$ [43-45, 72, 73]. These calculations, employing two reference structures on the same PES, represent the simplest way to judge the performance of $a b$ initio techniques for the calculation of semiglobal PESs. Indeed, they provide plentiful instruction in the pursuit of the $a b$ initio limit. Following Table VII of Ref. [43], the diverse (basis set, correlation) convergence may be characterized as follows: $\mathrm{NH}_{3}$ and $\mathrm{SiH}_{3}{ }^{-}$ inversion barriers and $\mathrm{H}_{2} \mathrm{O}$ barrier to linearity (poor, good); $\mathrm{HNCO}$ inversion barrier (good, poor); $\mathrm{C}_{2} \mathrm{H}_{6}$ and $\mathrm{HCOOH}$ torsional energetics (good, good); and $\mathrm{SiC}_{2}$ barrier to linearity (poor, poor).

In Table 1 we extend our previous valence-only focal-point studies [43-45, 72-74] with a problem in the (good, good) category, and report results for the rotational barrier of $\mathrm{CH}_{3} \mathrm{SiH}_{3}$, representing the difference between the energies of the eclipsed and staggered conformations. RHF theory recovers most of the rotational barrier; specifically, the RHF bar- 
Table 1. Valence focal-point analysis of the torsional barrier of methyl silane ${ }^{a}$

\begin{tabular}{|c|c|c|c|c|c|c|c|}
\hline Series CC & $\Delta E_{\mathrm{e}}(\mathrm{RHF})$ & $\delta[\mathrm{MP} 2]$ & $\delta[\mathrm{CCSD}]$ & $\delta[\mathrm{CCSD}(\mathrm{T})]$ & $\delta[\mathrm{CCSDT}]$ & $\delta[\mathrm{BD}(\mathrm{TQ})]$ & $\Delta E_{\mathrm{e}}(\mathrm{CC})$ \\
\hline cc-pVDZ(62) & 588 & +10 & -33 & +3 & -1 & -1 & 566 \\
\hline aug-cc-pVDZ(104) & 554 & -44 & -22 & -6 & -1 & {$[-1]$} & {$[480]$} \\
\hline cc-pVTZ(148) & 568 & -2 & -23 & 0 & {$[-1]$} & {$[-1]$} & [541] \\
\hline cc-pVTZ+d(153) & 577 & -3 & -22 & -1 & {$[-1]$} & {$[-1]$} & {$[549]$} \\
\hline CVTZ(210) & 577 & -10 & -22 & -1 & {$[-1]$} & {$[-1]$} & {$[542]$} \\
\hline aug-cc-pVTZ(234) & 576 & -28 & -23 & -4 & {$[-1]$} & {$[-1]$} & [519] \\
\hline aug-cc-pVTZ+d(239) & 583 & -27 & -23 & -4 & {$[-1]$} & {$[-1]$} & {$[527]$} \\
\hline cc-pVQZ(294) & 574 & -19 & -21 & -3 & {$[-1]$} & {$[-1]$} & {$[529]$} \\
\hline aug-cc-pVQZ(440) & 575 & -25 & {$[-21]$} & {$[-3]$} & {$[-1]$} & {$[-1]$} & {$[524]$} \\
\hline cc-pV5Z(516) & 579 & -22 & {$[-21]$} & {$[-3]$} & {$[-1]$} & {$[-1]$} & [531] \\
\hline aug-cc-pV5Z(738) & 579 & -26 & {$[-21]$} & {$[-3]$} & {$[-1]$} & {$[-1]$} & {$[527]$} \\
\hline CBS & 583 & -27 & -21 & -3 & -1 & -1 & 530 \\
\hline Series MP & $\Delta E_{\mathrm{e}}(\mathrm{RHF})$ & $\delta[\mathrm{MP} 2]$ & $\delta[\mathrm{MP} 3]$ & $\delta[\mathrm{MP} 4]$ & $\delta[\mathrm{MP} 5]$ & $\delta[\mathrm{MP} \infty]$ & $\Delta E_{\mathrm{e}}(\mathrm{MP})$ \\
\hline cc-pVDZ(62) & 588 & +10 & -23 & 0 & -5 & -1 & 568 \\
\hline aug-cc-pVDZ(104) & 554 & -44 & -18 & -4 & {$[-5]$} & {$[-1]$} & {$[482]$} \\
\hline cc-pVTZ(148) & 568 & -2 & -18 & -2 & {$[-5]$} & {$[-1]$} & {$[540]$} \\
\hline cc-pVTZ+d(153) & 577 & -3 & -17 & -2 & {$[-5]$} & {$[-1]$} & [549] \\
\hline aug-cc-pVTZ(234) & 576 & -28 & -19 & -4 & {$[-5]$} & {$[-1]$} & [519] \\
\hline aug-cc-pVQZ(440) & 575 & -25 & -17 & -4 & {$[-5]$} & {$[-1]$} & {$[523]$} \\
\hline
\end{tabular}

${ }^{a}$ For each basis set the total number of contracted Gaussian functions is given in parentheses. For correlated-level calculations the symbol $\delta$ denotes the increment in the relative energy $\left(\Delta E_{\mathrm{e}}\right)$ with respect to the preceding level of theory as given by the hierarchy RHF $\rightarrow$ MP2 $\rightarrow$ CCSD $\rightarrow$ $\mathrm{CCSD}(\mathrm{T}) \rightarrow \mathrm{CCSDT} \rightarrow \mathrm{BD}(\mathrm{TQ})$ and $\mathrm{RHF} \rightarrow \mathrm{MP} 2 \rightarrow \mathrm{MP} 3 \rightarrow \mathrm{MP} 4 \rightarrow \mathrm{MP} 5 \rightarrow \mathrm{MP} \infty$ for Series CC and Series MP, respectively. Brackets signify assumed increments from smaller basis set results. All values are given in $\mathrm{cm}^{-1}$. Extrapolations to the CBS limit were performed according to the formulas given in the text. 
Table 2. A comparison of predicted barriers to linearity for water $\left(\mathrm{H}_{2}^{16} \mathrm{O}\right.$ when adiabatic effects are considered)

\begin{tabular}{lll}
\hline Reference & Barrier Height & Comments \\
\hline Carter and Handy [78] & $11493 \mathrm{~cm}^{-1}$ & Spectroscopic Empirical \\
Jensen [79] & $11246 \mathrm{~cm}^{-1}$ & Spectroscopic Empirical \\
Polyansky et al. [80] & $10966 \mathrm{~cm}^{-1}$ & Spectroscopic Empirical \\
Lanquetin et al. $[81]$ & $11154 \mathrm{~cm}^{-1}$ & Effective Hamiltonian \\
Partridge and Schwenke (PS) [10] & $11155 \mathrm{~cm}^{-1}$ & Ab initio \\
Partridge and Schwenke [10] & $11128 \mathrm{~cm}^{-1}$ & Spectroscopic Empirical \\
PS + adiabatic + relativistic corr. & $11192 \mathrm{~cm}^{-1}$ & Ab initio \\
Császár et al. $[43]$ & $11046 \pm 70 \mathrm{~cm}^{-1}$ & Extrapolated ab initio \\
Tarczay et al. [44] & $11127 \pm 35 \mathrm{~cm}^{-1}$ & High accuracy ab initio \\
Kain et al. [82] & $11105 \pm 5 \mathrm{~cm}^{-1}$ & Semitheoretical \\
Valeev et al. [83] & $11119 \pm 12 \mathrm{~cm}^{-1}$ & Ab initio (MP2-R12) \\
\hline
\end{tabular}

rier is less than $55 \mathrm{~cm}^{-1}$ from the true valence-only electronic barrier. Since there are no stereochemically active lone pairs in $\mathrm{CH}_{3} \mathrm{SiH}_{3}$, diffuse functions are only moderately important. The valence-only CBS FCI barrier is $530 \mathrm{~cm}^{-1}$ (note the excellent agreement between extrapolated Series CC and Series MP results), as compared with the best experimental value of $603 \mathrm{~cm}^{-1}$ [75-77]. We estimate, at the CVTZ CCSD(T) level, the core-valence and the relativistic corrections as +6 and +1 $\mathrm{cm}^{-1}$, respectively. These small values are indicative of the lack of rehybridization during the rotational motion. These auxiliary corrections bring the calculated barrier to somewhat closer agreement with experiment; nevertheless, the remaining disrepancy deserves further studies.

In the various focal-point studies we looked critically at the auxiliary corrections to standard valence-only $a b$ initio electronic structure calculations. For water, for example, inclusion of the kinetic relativistic correction raises the all-electron extrapolated barrier height by $50 \mathrm{~cm}^{-1}$ or about $0.5 \%$. After this correction the extrapolated ab initio result for the barrier to linearity of water [43] was in good but not perfect agreement with the best empirical value available in 1998 [80]. In Table 2 we give a compilation of older and recent theoretical and empirical estimates of the barrier to linearity on the ground-state PES of water. Using the focal-point approach Császár et al. [43] deduced a converged estimate of $11046 \pm 70 \mathrm{~cm}^{-1}$ for the barrier, while later research [44], extending the previous work, determined $11127 \pm 35 \mathrm{~cm}^{-1}$. In an attempt to reproduce available empirical rovibrational levels supported by the ground electronic state of water, Kain et al. [82] modified existing highquality PESs $[10,22,27]$ and arrived at a value of $11105 \pm 5 \mathrm{~cm}^{-1}$ for the 
barrier, which is bracketed by the previous, nearly-converged ab initio estimates. Important conclusions of the first $a b$ initio investigations were that, as usual, it was easiest to estimate the Hartree-Fock (HF) limit, the well-established HF value being $11247 \pm 2 \mathrm{~cm}^{-1}$; it proved hardest to arrive at the MP2 limit; and corrections beyond MP2 were much less dependent on the quality of the basis set. Using the MP2-R12/A level and very large basis sets, Valeev et al. [83] have most recently obtained a converged MP2 estimate of $-357 \pm 5 \mathrm{~cm}^{-1}$, significantly different from the best previous $a b$ initio estimate of $-348 \mathrm{~cm}^{-1}$ [44]. The improved estimate of the MP2 limit yielded an improved (lower) estimate for the uncertainty of the $a b$ initio barrier. Relativistic corrections beyond the kinetic term also influence the barrier. For example, the Gaunt correction increases the barrier by $6 \mathrm{~cm}^{-1}$, while consideration of the Lambshift effect [29] lowers the barrier by almost $4 \mathrm{~cm}^{-1}$. These and some other high-quality $a b$ initio results led Valeev et al. [83] to surmise that the best present $a b$ initio estimate of the barrier to linearity of $\mathrm{H}_{2} \mathrm{O}$ is $11119 \pm 12 \mathrm{~cm}^{-1}$. In summary, consideration of all possible computational effects on the barrier has finally resulted in excellent accord between theory and experiment.

\section{SCALED HIGHER-ORDER CORRELATION ENERGIES (SHOC)}

Since FCI computations are very expensive, even with small basis sets, there is little hope that larger basis set FCI calculations will become routine in the near future. Even with the explosive growth in computing power, FCI benchmarks are typically limited to small basis sets and molecules with up to two heavy atoms $[60,85-96]$. However, large basis set coupled-cluster (CC) computations, which include through triple excitations (CCSD $(\mathrm{T})[56]$ and $\operatorname{CCSDT}[57,58])$, are viable for a large number of molecular systems where small basis set FCI computations are feasible.

Therefore, in a recent study Császár and Leininger [62] explored a simple multiplicative approach, termed scaled higher-order correlation (SHOC), for approximating the full configuration interaction (FCI) limit at the complete basis set (CBS) limit from small basis set FCI and coupled cluster (most notably $\operatorname{CCSD}(\mathrm{T})$ and CCSDT) calculations. In the SHOC approach an attempt is made to scale correlation energies obtained at lower levels of theory to correct for neglected excitations and arrive at the FCI limit within a particular one-particle basis set. Note that linear scalings, though with different goals in mind, have been employed before, see, e.g., the PCI-X method of Siegbahn and co-workers 
Table 3. Hartree-Fock and correlation energies (in $E_{\mathrm{h}}$ ) of the $\mathrm{N}\left({ }^{4} S\right)$ atom and the $\mathrm{N}_{2}$ molecule (at $r=1.0996 \AA$ ) calculated at different levels of theory, and the resulting valence-only total atomization energy (TAE, in $\mathrm{kcal} / \mathrm{mol}$ ) of $\mathrm{N}_{2}^{a}$

\begin{tabular}{lcccc}
\hline & $(\mathrm{U}) \mathrm{HF}$ & $\Delta E_{\text {corr }}[(\mathrm{U}) \mathrm{CCSD}]$ & $\Delta E_{\text {corr }}[(\mathrm{U}) \mathrm{CCSDT}]$ & $\Delta E_{\text {corr }}[\mathrm{FCI}]$ \\
\hline $\mathrm{N}$ atom & & & & \\
cc-pVDZ & -54.391115 & -0.086760 & -0.087517 & -0.087436 \\
cc-pVTZ & -54.400686 & -0.111746 & -0.114341 & -0.114183 \\
cc-pVQZ & -54.403718 & -0.118297 & -0.121440 & -0.121294 \\
CBS & -54.405124 & -0.123077 & -0.126620 & -0.126483 \\
$\mathrm{~N}_{2}$ & & & & \\
cc-pVDZ & -108.953856 & -0.309691 & -0.321684 & -0.323350 \\
cc-pVTZ & -108.983089 & -0.372249 & -0.390516 & -0.392539 \\
cc-pVQZ & -108.990687 & -0.393463 & -0.413286 & -0.415426 \\
CBS & -108.993355 & -0.408943 & -0.429902 & -0.432127 \\
\hline TAE & $\mathrm{HF}$ & $\mathrm{CCSD}$ & $\mathrm{CCSDT}$ & FCI \\
\hline cc-pVDZ & 107.7 & 193.1 & 200.3 & 200.9 \\
cc-pVTZ & 114.0 & 207.4 & 215.6 & 217.0 \\
cc-pVQZ & 115.0 & 213.4 & 221.9 & 223.4 \\
CBS & 114.9 & 217.1 & 225.8 & 227.3 \\
\hline
\end{tabular}

${ }^{a}$ Finite-basis HF, CCSD, CCSDT, and FCI results are taken from Refs. [101, 102]. The FCI results reported for $\mathrm{N}_{2}$ utilize CCSDT results and the SHOC scheme (see text). Basis set extrapolations were performed with formulas given in the text.

[97], the G3S method of Pople and co-workers [38], as well as the scaled external correlation (SEC) scheme [98], and the scaling all correlation (SAC) method [99] of Truhlar and co-workers. The utility of the HOC scaling approach is best understood by examples. In this review two examples are given. The first concerns the total atomization energy of $\mathrm{N}_{2}$. The second example concerns the PES of $\mathrm{H}_{2} \mathrm{O}$.

\subsection{HOC EFFECTS ON THE TOTAL ATOMIZATION ENERGY OF $\mathrm{N}_{2}$}

The scaled higher-order correlation (SHOC) correction scheme has been demonstrated [62] to extrapolate $\operatorname{CCSD}(\mathrm{T})$ correlation energies for the global minima of the ground electronic states of $\mathrm{BH}$ and $\mathrm{AlH}$, as well as $\tilde{\mathrm{X}}^{3} \mathrm{~B}_{1}$ and $\tilde{\mathrm{a}}^{1} \mathrm{~A}_{1} \mathrm{CH}_{2}$ [100], to the FCI limit with almost $\mu E_{\mathrm{h}}$ accuracy. The SHOC approach is used here to investigate the HOC correction on the total atomization energy of $\mathrm{N}_{2}$, employing data computed at the CCSD, CCSDT, and FCI levels by Feller and Sordo [101-103]. As it is clear from Table 5.1, containing Hartree-Fock and correlation energies for the $\mathrm{N}\left({ }^{4} S\right)$ atom and the $\mathrm{N}_{2}$ molecule, the SHOC correction 
for the total atomization energy of $\mathrm{N}_{2}$, with a SHOC factor of 1.005182 obtained from our own cc-pVDZ CCSDT and FCI computations, is +1.5 $\mathrm{kcal} / \mathrm{mol}$. The resulting extrapolated CBS FCI value is $227.3 \mathrm{kcal} / \mathrm{mol}$. Core-valence correlation and relativistic effects, not considered during calculation of the valence-only CBS FCI value, have been estimated by Feller and Sordo [102] to be $+0.8 \mathrm{kcal} / \mathrm{mol}$. This last correction brings our computed value very close to the best experimental value, $228.5 \pm 0.4 \mathrm{kcal} / \mathrm{mol}[104]$.

\subsection{HOC EFFECTS ON POTENTIAL ENERGY SURFACES}

The proposed SHOC scheme is multiplicative, and it can readily be applied to the study of PESs. The effectiveness of using SHOC has been tested on the ground-state $\mathrm{PES}$ of $\mathrm{H}_{2} \mathrm{~S}$ in Refs. $[62,71]$. The HOC energy corrections resulted in an order of magnitude uniform reduction of the valence-only correlation energy error of the CCSD(T) calculation. Here the analysis is extended to $\mathrm{H}_{2} \mathrm{O}$. Four geometries [105] have been selected, covering an energy range of $0-23000 \mathrm{~cm}^{-1}$. Valenceonly cc-pVDZ FCI and CCSD(T) computations have been performed at these geometries, resulting in estimates of valence-only correlation energies. The FCI - CCSD $(\mathrm{T})$ energy differences before and after the HOC scaling, using the arithmetical average of the scale factors determined, 1.002811, are as follows: $[-636,-625,-706,-419]$ and $[-34,-26$, $-97,+147] \mu E_{\mathrm{h}}$, respectively. It is clear from these numbers that the SHOC scale factors change relatively little over the PES and that the SHOC correction results again in an order of magnitude reduction in the valence-only correlation energy error of the $\operatorname{CCSD}(\mathrm{T})$ calculation.

\section{THE VIBRATIONAL SPECTRUM OF $\mathrm{H}_{2} \mathrm{~S}$}

Several valence-only potential energy hypersurfaces have been determined at the CCSD(T) level for $\mathrm{H}_{2} \mathrm{~S}$ in Ref. [71], with aug-cc-pVTZ+d, aug-cc-pVQZ+d, and aug-cc-pV5Z basis sets. As discussed in some detail in Refs. [45, 106], augmentation of the original aug-cc-pVXZ basis sets of Dunning $[52,53]$ for $\mathrm{S}$ with tight polarization functions $(+\mathrm{d})$ is necessary in order to obtain reliable RHF energies. This so-called core polarization effect has been dealt with through extension of the $d$-space $(+\mathrm{d})$ of the aug-cc-pVXZ $(X=\mathrm{T}$ and $\mathrm{Q})$ basis sets with an additional function whose exponent is the same as the largest $d$ exponent in the aug-cc-pV5Z basis. Another surface, denoted CBS CCSD(T), results when the electronic energies obtained with the finite basis sets are extrapolated, using the equations given in Section 2, to the complete basis 
Table 4. Differences between observed (obs.) vibrational band origins of $\mathrm{H}_{2}^{32} \mathrm{~S}$ and those computed at the $\operatorname{CCSD}(\mathrm{T}) \operatorname{level}^{a}$

\begin{tabular}{lrrrrrr}
\hline Label & Obs. & TZ+d & QZ+d & $5 Z$ & CBS & CBS FCI \\
\hline $010 / 001$ & 1182.57 & -0.3 & -1.7 & -2.1 & -2.5 & -3.0 \\
$020 / 002$ & 2353.96 & +5.4 & +2.2 & +0.9 & +0.3 & -0.7 \\
$100 / 10^{+} 0$ & 2614.14 & -5.0 & +0.9 & +1.8 & +3.1 & +2.3 \\
$001 / 10^{-} 0$ & 2628.46 & -6.1 & +0.2 & +1.3 & +2.5 & +1.8 \\
$030 / 003$ & 3513.79 & +11.3 & +6.3 & +4.1 & +3.5 & +1.9 \\
$040 / 004$ & 4661.68 & +14.3 & +7.6 & +5.1 & +4.2 & +2.2 \\
$120 / 10^{+} 2$ & 4932.70 & +2.1 & +5.0 & +4.2 & +5.0 & +3.2 \\
$021 / 10^{-} 2$ & 4939.10 & -0.2 & +3.4 & +3.6 & +4.4 & +2.6 \\
$200 / 20^{+} 0$ & 5144.99 & -10.2 & +2.9 & +4.6 & +6.9 & +5.4 \\
$101 / 20^{-} 0$ & 5147.22 & -10.8 & +2.5 & +4.0 & +6.5 & +5.0 \\
$002 / 110$ & 5243.10 & -10.7 & +1.6 & +3.6 & +5.8 & +4.4 \\
$050 / 005$ & 5797.24 & +12.8 & +4.7 & +2.8 & +1.5 & -1.1 \\
$210 / 20^{+} 1$ & 6288.15 & -9.4 & +2.7 & +3.9 & +5.8 & +3.7 \\
$111 / 20^{-} 1$ & 6289.17 & -10.0 & +2.2 & +3.4 & +5.5 & +3.5 \\
$300 / 30^{+} 0$ & 7576.38 & -16.4 & +5.3 & +7.8 & +11.4 & +9.0 \\
$102 / 21^{+} 0$ & 7752.26 & -16.2 & +5.4 & +8.0 & +11.5 & +9.0 \\
$003 / 21^{-} 0$ & 7779.32 & -16.2 & +3.4 & +6.3 & +9.5 & +7.2 \\
\hline
\end{tabular}

${ }^{a}$ All values are given in $\mathrm{cm}^{-1}$. All differences are reported as calculated - observed. $\mathrm{TZ}+\mathrm{d}$ $=$ aug-cc-pVTZ + d CCSD $(\mathrm{T})$ PES; QZ + d = aug-cc-pVQZ + d CCSD $(\mathrm{T})$ PES; $5 \mathrm{Z}=$ augcc-pV5Z CCSD(T) PES; CBS = complete basis set CCSD(T) PES; CBS FCI = CBS full configuration interaction PES. Normal mode $\left(v_{1} v_{2} v_{3}\right) /$ local mode $\left(v_{r 1} v_{r 2} v_{\theta}\right)$ labeling. Observed VBOs from Ref. [107]. Average absolute errors, in $\mathrm{cm}^{-1}$, for the entries of this table are as follows: $\mathrm{TZ}+\mathrm{d}=9.3, \mathrm{QZ}+\mathrm{d}=3.4,5 \mathrm{Z}=4.0, \mathrm{CBS}=5.3$, and $\mathrm{CBS} \mathrm{FCI}=3.9$.

set (CBS) limit. The most accurate valence-only PES, at least within the present approach, is denoted as CBS FCI and is obtained when the CBS CCSD $(\mathrm{T})$ correlation energies are scaled, as described in Section 5, to the FCI limit. All these surfaces have been employed to calculate VBOs for $\mathrm{H}_{2}^{32} \mathrm{~S}$. The results obtained are presented in Table 4.

It is clear from Table 4 that extension of the basis set $(X=3,4,5$, and $\infty)$ shifts the calculated VBOs more or less systematically. The least accurate results are obtained with $X=3$; however, the CBS CCSD(T) results, though substantially different from the $X=3$ results, deviate about as much from experiment. The $X=4$ results [aug-cc-pVQZ+d $\operatorname{CCSD}(\mathrm{T})]$ have the smallest mean deviation. This observation is not surprising, in fact it is a result of fortuitious error cancellation which is often utilized in quantum chemical calculations. Much better agreement between theory and experiment is achieved when core-valence correlation, relativistic, and DBOC effects are included in the $a b$ initio treatment [71]. 


\section{THE ROVIBRATIONAL SPECTRUM OF $\mathrm{H}_{2} \mathrm{O}$}

There is a very high quality $a b$ initio ground-state PES available for $\mathrm{H}_{2} \mathrm{O}$, determined by Partridge and Schwenke [10]. Nevertheless, neither basis set extrapolations nor HOC energy corrections have been performed to reach the CBS FCI limit. For this reason and due to remaining computational errors, small empirical adjustment [10] of the ab initio PES resulted in a much better quality semitheoretical PES, which reproduces a large number of rovibrational levels up to about $10000 \mathrm{~cm}^{-1}$ with an average accuracy of about $0.2 \mathrm{~cm}^{-1}$, and levels up to 18000 $\mathrm{cm}^{-1}$ with an average accuracy of about $1.2 \mathrm{~cm}^{-1}$. From the detailed $a b$ initio studies $[43,44]$ of the barrier to linearity of water, it became clear that relativistic corrections should influence substantially the calculated VBOs. Over the last two years we calculated several relativistic correction surfaces for the ground-state PES of $\mathrm{H}_{2} \mathrm{O}$, including a one-electron mass-velocity and Darwin (MVD1) surface [27], a two-electron Darwin (D2) surface, a Gaunt correction surface, and correction surfaces due to the Lamb-shift effect [29].

Our results, presented in Tables 5 and 6 , clearly show that the relativistic corrections have a significant influence on the calculated behavior of both the vibrational and rotational states of water. This should be compared with inclusion of the DBOC, which only has a minor influence [22]. As expected from the changes in the barrier to linearity found upon inclusion of relativistic effects $[43,44,29]$, the relavistic corrections to the PES can either raise or lower the rovibrational bands. For example, the kinetic relativistic effect (MVD1) raises both the barrier and the band origins of the bending states. Empirical test calculations [82] which augmented the $\mathrm{BO}$ potential with a simple term proportional to the bending coordinate alone resulted in a decrease in the barrier height and a simultaneous decrease in bending band origins. These observations are particularly interesting because of difficulties encountered in representing bending excitations both in water [80] and in $\mathrm{H}_{2} \mathrm{~S}$ [107] by fitting to spectroscopic data. While the average discrepancy between $a b$ initio theory and observation for the VBOs of water is not significantly changed by inclusion of the relativistic corrections in the calculation, there is a marked shift in the error. The error in all band origins using the nonrelativistic BO potential surface of Partridge and Schwenke [10] is approximately constant at $0.1-0.2 \%$. Inclusion of the relativistic correction in the PES greatly improves predictions for the stretching states at the expense of worsening (doubling) the error for the pure bending modes. It is also clear from the calculated VBOs that, in accord with 
Table 5. Independent contributions of selected relativistic correction surfaces to the vibrational $(J=0)$ band origins of $\mathrm{H}_{2}^{16} \mathrm{O}^{a}$

\begin{tabular}{crrrrrr}
\hline$\left(v_{1}, v_{2}, v_{3}\right)$ & Observed & +MVD1 & +D2 & +Gaunt & +L1 & +L2 \\
\hline$(010)$ & 1594.75 & +1.29 & +0.09 & -0.09 & -0.09 & +0.00 \\
$(020)$ & 3151.63 & +2.73 & +0.18 & -0.15 & -0.18 & +0.01 \\
$(030)$ & 4666.79 & +4.38 & +0.28 & -0.17 & -0.29 & +0.02 \\
$(040)$ & 6134.01 & +6.40 & +0.40 & -0.13 & -0.43 & +0.02 \\
$(050)$ & 7542.44 & +8.96 & +0.54 & -0.01 & -0.60 & +0.03 \\
$(060)$ & 8869.95 & +12.72 & +0.73 & +0.27 & -0.86 & +0.04 \\
& & & & & & \\
$(100)$ & 3657.05 & -2.80 & -0.05 & -0.79 & +0.18 & +0.00 \\
$(200)$ & 7201.54 & -5.60 & -0.09 & -1.57 & +0.36 & -0.01 \\
$(300)$ & 10599.69 & -8.38 & -0.14 & -2.34 & +0.54 & -0.01 \\
$(400)$ & 13828.28 & -11.06 & -0.18 & -3.09 & +0.71 & -0.01 \\
$(500)$ & 16898.40 & -13.0 & -0.20 & -3.73 & +0.83 & -0.01 \\
$(600)$ & 19782.00 & & -0.26 & -4.47 & +1.01 & -0.01 \\
$(700)$ & 22529.30 & & -0.34 & & +1.19 & -0.02 \\
& & & & & & \\
$(101)$ & 7249.82 & -5.68 & -0.09 & -1.69 & +0.37 & -0.01 \\
$(201)$ & 10613.35 & -8.43 & -0.14 & -2.31 & +0.54 & -0.01 \\
$(301)$ & 13830.94 & -11.05 & -0.18 & -1.83 & +0.71 & -0.01 \\
$(401)$ & 16898.84 & -12.98 & -0.20 & -4.66 & +0.83 & -0.01 \\
$(501)$ & 19781.10 & -15.80 & -0.26 & & +1.01 & -0.01 \\
$(601)$ & 22529.44 & -19.31 & -0.34 & & +1.19 & -0.02 \\
$(701)$ & 25120.28 & & -0.39 & & +1.29 & -0.02 \\
\hline
\end{tabular}

${ }^{a}$ All VBOs and corrections are given in $\mathrm{cm}^{-1}$. Observed VBOs, provided only for guidance, are taken from Refs. [108, 109, 110]. The one-electron mass-velocity and Darwin (MVD1) results are taken from Ref. [27]. L1 = one-electron Lamb-shift correction surface. L2 = twoelectron Lamb-shift correction surface. The Lamb-shift correction surfaces were obtained using the procedure of Ref. [29].

expectation, the kinetic relativistic correction has the largest effect followed by the Gaunt correction and the Lamb shift. Note that the full Breit correction [28] is somewhat smaller than the Gaunt correction. It is also notable how small the influence of the 2-electron correction terms (2electron Darwin, D2, and 2-electron Lamb, L2) is on the VBOs, though for some VBOs, especially for the bends, the D2 correction, increasing with increasing excitation, is not negligible.

Table 6 shows the $J=20$ rotational term values calculated using the same models analysed above for the VBOs. Results are only presented for the vibrational ground state [109]. The effect of the inclusion of relativistic corrections on the rotational term values is strongly dependent on $K_{a}$. For high values of $K_{a}$, the relativistic correction lowers the rotational term values leading to significant disagreement with the observed 
Table 6. Independent contributions of selected relativistic correction surfaces to the $J=20$ rotational $\left[\left(v_{1} v_{2} v_{3}\right)=(000)\right]$ energy levels of $\mathrm{H}_{2}^{16} \mathrm{O}^{a}$

\begin{tabular}{lcrrrrr}
\hline$J_{K_{a}, K_{c}}$ & Observed & +MVD1 & $+\mathrm{D} 2$ & + Gaunt & $+\mathrm{L} 1$ & $+\mathrm{L} 2$ \\
\hline $20_{0,20}$ & 4048.250 & -0.455 & +0.067 & -1.051 & +0.079 & +0.003 \\
$20_{1,19}$ & 4412.316 & -0.481 & +0.073 & -1.144 & +0.085 & +0.004 \\
$20_{2,18}$ & 4738.622 & -0.426 & +0.083 & -1.214 & +0.085 & +0.004 \\
$20_{3,17}$ & 5031.794 & -0.297 & +0.094 & -1.268 & +0.078 & +0.006 \\
$20_{4,16}$ & 5292.103 & -0.045 & +0.111 & -1.297 & +0.064 & +0.006 \\
$20_{5,15}$ & 5513.236 & +0.526 & +0.141 & -1.282 & +0.027 & +0.007 \\
$20_{6,14}$ & 5680.788 & +1.400 & +0.183 & -1.218 & -0.032 & +0.009 \\
$20_{7,13}$ & 5812.074 & +1.397 & +0.186 & -1.247 & -0.032 & +0.010 \\
$20_{8,12}$ & 5966.823 & +0.206 & +0.137 & -1.421 & +0.052 & +0.007 \\
$20_{9,11}$ & 6170.832 & -1.023 & +0.087 & -1.609 & +0.140 & +0.004 \\
$20_{10,10}$ & 6407.443 & -1.968 & +0.051 & -1.771 & +0.207 & +0.002 \\
$20_{11,9}$ & 6664.173 & -2.820 & +0.018 & -1.924 & +0.268 & +0.001 \\
$20_{12,8}$ & 6935.428 & -3.646 & -0.013 & -2.075 & +0.328 & -0.001 \\
$20_{13,7}$ & 7217.562 & -4.463 & -0.043 & -2.226 & +0.386 & -0.002 \\
$20_{14,6}$ & 7507.545 & -5.274 & -0.072 & -2.375 & +0.445 & -0.005 \\
$20_{15,5}$ & 7802.709 & -6.084 & -0.103 & -2.523 & +0.502 & -0.005 \\
$20_{16,4}$ & 8100.291 & -6.895 & -0.132 & -2.669 & +0.560 & -0.007 \\
$20_{17,3}$ & 8397.648 & -7.712 & -0.162 & -2.814 & +0.617 & -0.009 \\
$20_{18,2}$ & 8691.927 & -8.538 & -0.193 & -2.956 & +0.676 & -0.011 \\
$20_{19,1}$ & 8979.881 & -9.382 & -0.225 & -3.095 & +0.735 & -0.012 \\
$20_{20,0}$ & 9257.459 & -10.260 & -0.258 & -3.230 & +0.797 & -0.015 \\
\hline$a$
\end{tabular}

${ }^{a}$ All rotational data are given in $\mathrm{cm}^{-1}$. The observed rotational term values, provided only for guidance, are taken from Refs. [108, 109, 111, 112]. Many rotational transitions come in quasi-degenerate pairs (cf. Ref. [27]). To save space, only one member in each pair is reported in this table. The one-electron mass-velocity and Darwin (MVD1) results are taken from Ref. [27]. L1 = one-electron Lamb-shift correction surface. L2 $=$ two-electron Lamb-shift correction surface. The Lamb-shift correction surfaces were obtained using the procedure of Ref. [29].

levels [27]. This is consistent with the large increase observed in the VBOs of the bending overtones. Similarly to the VBOs, the order of the magnitude of the different corrections to the rotational term values is kinetic $>$ Gaunt $>$ Lamb. The two-electron relativistic corrections (D2 and L2) are again rather small, the two-electron Lamb-shift correction can safely be ignored. This is not true, however, for the one-electron Lamb shift, L1. For example, the $20_{20,0}$ rotational level is shifted, due to L1, by more than $0.7 \mathrm{~cm}^{-1}$, some 500 times more than the present experimental accuracy [108], which in this case could be improved by up to three orders of magnitude using current technology. The Lamb-shift effect increases with increasing excitation both for the vibrations and for the rotations. 


\section{SUMMARY}

High-resolution rovibrational spectra provide a wealth of information on molecular properties and vibration-rotation dynamics provided that the "inverse eigenvalue problem" of molecular spectroscopy can be unravelled to some degree. First-principles techniques do not suffer from the inverse eigenvalue dilemma and thus provide both complementary and competitive approaches to the understanding of chemical phenomena. A big step in this direction is the ab initio determination of potential energy hypersurfaces of "spectroscopic" accuracy. In this review a snapshot of some aspects of the state of evolution of $a b$ initio methodologies in the determination of accurate PESs has been presented. The focus of the attention has been on the focal-point approach involving a dual extrapolation to the complete basis set (CBS) and full configuration interaction (FCI) asymptotes. The numerical results presented show again the intrinsic accuracy and utility of $a b$ initio techniques in general and the focal-point approach in particular. It is shown that scaling the higher-order correlation energy (SHOC) increases the dissociation energy, $D_{\mathrm{e}}$, of $\mathrm{N}_{2}$, and reduces the error with respect to experiment, and it also results in an order of magnitude error reduction in the FCI $\operatorname{CCSD}(\mathrm{T})$ correlation energy of $\mathrm{H}_{2} \mathrm{O}$. A pragmatic and sophisticated $a b$ initio approach, based on the focal-point and SHOC schemes, has been utilized for the construction of valence-only PESs for the ground electronic state of $\mathrm{H}_{2} \mathrm{~S}$. Convergence analysis of the VBOs computed using these PESs reveals the usefulness of the approach. Small corrections, not considered in the valence-only treatment, including core-valence correlation, relativistic effects, and the diagonal Born-Oppenheimer correction (DBOC), are known to be important for the accurate prediction of rovibrational levels. In this review the relativistic effects are quantified for the rovibrational spectrum of $\mathrm{H}_{2} \mathrm{O}$. It is shown that among the relativistic effects the dominant one is the kinetic relativistic effect, while, in general, the Gaunt correction and quantum electrodynamics (QED) corrections make smaller and smaller contributions to the rovibrational levels. Nevertheless, at about $10000 \mathrm{~cm}^{-1}$ and above the QED effect may result in changes as large as $1 \mathrm{~cm}^{-1}$ for some rovibrational states.

\section{Acknowledgments}

The work of Attila G. Császár and György Tarczay has been supported by the Hungarian Ministry of Culture and Education (FKFP 0117/1997) and by the Scientific Research Fund of Hungary (OTKA T024044 and T033074). The work of Matthew L. Leininger was supported by Sandia National Laboratories. Sandia is a multiprogram lab- 
oratory operated by Sandia Corporation, a Lockheed Martin Company, for the United States Department of Energy under Contract DE-AC0494AL85000. The work of Jonathan Tennyson and Oleg L. Polyansky was supported by the UK Engineering and Physical Science Research Council (grant GR/K47702). The work of Oleg L. Polyansky was also supported by the Russian Fund for Fundamental Studies. Scientific exchanges between Budapest and London received support from the Hungarian-British Joint Academic and Research Programme (project no. 076). Scientific exchanges between Budapest and Athens, GA received support from a NATO Linkage Grant (CRG.LG 973892).

\section{References}

[1] Oka, T. (1980) Phys. Rev. Lett., 45, 531-534.

[2] Geballe, T.R. (2000) Phil. Trans. Royal Soc. London A, 358, 2503-2512.

[3] Miller, S., Tennyson, J., Lepp, S. and Dalgarno, A. (1992) Nature, 355, 420422.

[4] Miller, S., Achilleos, N., Ballester, G.E., Geballe, T.R., Joseph, R.D., Prange, R., Rego, D., Stallard, T.S., Tennyson, J., Trafton, L.M. and Waite Jr., J.H. (2000) Phil. Trans. Royal Soc. London A, 358, 2485-2501.

[5] Kao, L., Oka, T., Miller, S. and Tennyson, J. (1991) Astrophys. J. Suppl., 77, 317-329.

[6] The Opacity Project Team (1995) The Opacity Project, vol. 1 and (1996) The Opacity Project, vol. 2, IOP Publishing: Bristol, UK.

[7] Allard, F., Hauschildt, P. H., Alexander, D. R. and Starrfield, S. (1997) Ann. Rev. Astron. Astrophys., 35, 137-177.

[8] Jorgensen, U.G., Almlöf, J., Gustafsson, B., Larsson, M. and Siegbahn, P. (1986) J. Chem. Phys., 83, 3034-3042.

[9] Allard, F., Hauschildt, P.H., Miller, S. and Tennyson, J. (1994) Astrophys. J., 426, L39-L41.

[10] Partridge, H. and Schwenke, D.W. (1997) J. Chem. Phys, 106, 4618-4639.

[11] Allard, F., Hauschildt, P.H. and Schwenke, D.W. (2000) Astrophys. J., 540, 1005-1015.

[12] Neale, L. and Tennyson, J. (1995) Astrophys. J., 454, L169-L173.

[13] Sidhu, K.S., Miller, S. and Tennyson, J. (1992) Astron. Astrophys., 255, 453456.

[14] Ramanathan, V. and Vogelmann, A.M. (1997) Ambio, 26, 38-46.

[15] Polyansky, O.L., Zobov, N.F., Viti, S., Tennyson, J. Bernath, P.F. and Wallace, L. (1997) Science, 277, 346-348.

[16] Polyansky, O.L., Zobov, N.F., Viti, S., Tennyson, J., Bernath, P.F. and Wallace, L. (1997) J. Molec. Spectrosc., 186, 422-447.

[17] Kedziora, G.S. and Shavitt, I. (1997) J. Chem. Phys., 106, 8733-8745.

[18] Császár, A.G. and Mills, I.M. (1997) Spectrochimica Acta, 53A, 1101-1122. 
[19] Császár, A.G., Allen, W.D., Yamaguchi, Y. and Schaefer III, H.F. (2000) in Computational Molecular Spectroscopy, Eds. P. Jensen and P. R. Bunker, Wiley: New York.

[20] Searles, D. and Nagy-Felsobuki, E. (1993) Ab Initio Variational Calculations of Molecular Vibrational-Rotational Spectra, Springer-Verlag: Berlin.

[21] Tennyson, J. (2000) in Computational Molecular Spectroscopy, Eds. P. Jensen and P. R. Bunker, Wiley: New York.

[22] Zobov, N.F., Polyansky, O.L., Le Sueur, C.R. and Tennyson, J. (1996) Chem. Phys. Lett., 260, 381-387.

[23] Balasubramanian, K. (1997) Relativistic Effects in Chemistry, Part A: Theory and Techniques and Part B: Applications, Wiley: New York.

[24] Cencek, W., Rychlewski, J., Jaquet, R. and Kutzelnigg, W. (1998) J. Chem. Phys., 108, 2831-2836.

[25] Jaquet, R., Cencek, W., Kutzelnigg, W. and Rychlewski, J. (1998) J. Chem. Phys., 108, 2837-2846.

[26] Polyansky, O.L. and Tennyson, J. (1999) J. Chem. Phys., 110, 5056-5064.

[27] Császár, A.G., Kain, J.S., Polyansky, O.L., Zobov, N.F. and Tennyson, J. (1998) Chem. Phys. Lett., 293, 317-323; (1999) ibid, 312, 613-616 (E).

[28] Quiney, H.M., Barletta, P., Tarczay, G., Császár, A.G., Polyansky, O.L. and Tennyson, J. (2001) Chem. Phys. Lett. to be submitted.

[29] Pyykkö, P., Dyall, K.G., Császár, A.G., Tarczay, G., Polyansky, O.L. and Tennyson, J. (2000) Phys. Rev. A in print.

[30] Tarczay, G., Császár, A.G. and Klopper, W. (2001) J. Chem. Phys. to be submitted.

[31] Murrell, J.N., Carter, S., Farantos, S.C., Huxley, P. and Varandas, A.J.C. (1984) Molecular Potential Energy Surfaces, Wiley: New York.

[32] Hirst, D.M. (1985) Potential Energy Surfaces, Taylor and Francis: London.

[33] Born, M. and Oppenheimer, J.R. (1927) Ann. Physik, 84, 457.

[34] Born, M. and Huang, K. (1954) Dynamical Theory of Crystal Lattices, Oxford University Press: London.

[35] Curtiss, L.A. and Raghavachari, K. (1998) ACS Symp. Ser., 677, 176.

[36] Curtiss, L.A., Raghavachari, K., Trucks, G.W. and Pople, J.A. (1991) J. Chem. Phys., 94, 7221-7230.

[37] Curtiss, L.A., Raghavachari, K., Redfern, P.C., Rassolov, V. and Pople, J.A. (1998) J. Chem. Phys., 109, 7764-7776.

[38] Curtiss, L.A., Raghavachari, K., Redfern, P.C. and Pople, J.A. (2000) J. Chem. Phys., 112, 1125-1132.

[39] Montgomery Jr., J.A., Ochterski, J.W. and Petersson, G.A. (1994) J. Chem. Phys., 101, 5900-5909.

[40] Montgomery Jr., J.A., Frisch, M.J., Ochterski, J.W. and Petersson, G.A. (1999) J. Chem. Phys., 110, 2822-2827.

[41] Martin, J.M.L. and Oliveira, G. (1999) J. Chem. Phys., 111, 1843-1856. 
[42] Allen, W.D., East, A.L.L. and Császár, A.G. (1993) in Structures and Conformations of Non-Rigid Molecules, NATO ASI Series C, Eds. J. Laane, M. Dakkouri, B. van der Veken, and H. Oberhammer, Kluwer: Dordrecht, p.343.

[43] Császár, A.G., Allen, W.D. and Schaefer III, H.F. (1998) J. Chem. Phys., 108, 9751-9764.

[44] Tarczay, G., Császár, A.G., Klopper, W.M., Szalay, V., Allen, W.D. and Schaefer III, H.F. (1999) J. Chem. Phys., 110, 11971-11981.

[45] Tarczay, G., Császár, A.G., Leininger, M.L. and Klopper, W.M. (2000) Chem. Phys. Lett., 322, 119-128.

[46] Klopper, W.M., Bak, K.L., Jørgensen, P., Olsen, J. and Helgaker, T. (1999) J. Phys. B, 32, 103-130.

[47] Feller, D. (1992) J. Chem. Phys., 96, 6104-6114.

[48] Martin, J.M.L. (1996) Chem. Phys. Lett., 259, 679-682.

[49] Halkier, A., Helgaker, T., Jørgensen, P., Klopper, W.M. and Olsen, J. (1999) Chem. Phys. Lett., 302, 437-446.

[50] Halkier, A., Helgaker, T., Klopper, W.M. and Olsen, J. (2000) Chem. Phys. Lett., 319, 287-295.

[51] Halkier, A., Helgaker, T., Klopper, W., Jørgensen, P. and Császár, A.G. (1999) Chem. Phys. Lett., 310, 385-389.

[52] Dunning Jr., T.H. (1989) J. Chem. Phys., 90, 1007-1023.

[53] Kendall, R.A., Dunning Jr., T.H. and Harrison, R.J. (1992) J. Chem. Phys., 96, 6796-6806.

[54] Wilson, K.A., v. Mourik, T. and Dunning Jr., T.H., (1997) J. Mol. Struct. (THEOCHEM), 338, 339-349.

[55] Bartlett, R.J. (1997) Recent Advances in Coupled Cluster Methods, World Scientific: Singapore.

[56] Raghavachari, K., Trucks, G.W., Pople, J.A. and Head-Gordon, M. (1989) Chem. Phys. Lett., 157, 479-483.

[57] Noga, J. and Bartlett, R.J. (1987) J. Chem. Phys., 86, 7041-7050; (1988) ibid., 89, 3401(E).

[58] Scuseria, G. and Schaefer III, H.F. (1988) Chem. Phys. Lett., 152, 382-386.

[59] Sherrill, C.D. and Schaefer III, H.F. (1999) Adv. Quant. Chem., 34, 143-269.

[60] Leininger, M.L., Allen, W.D., Schaefer III, H.F. and Sherrill, C.D. (2000) J. Chem. Phys., 112, 9213-9222.

[61] Lee, T.J. and Scuseria, G.E. (1995) in Quantum Mechanical Electronic Structure Calculations with Chemical Accuracy, Ed. Langhoff, S.R., Kluwer: Dordrecht, pp. 47-108.

[62] Császár, A.G. and Leininger, M.L. (2001) J. Chem. Phys. submitted for publication.

[63] PSi 3.0, T. D. Crawford, C. D. Sherrill, E. F. Valeev, J. T. Fermann, M. L. Leininger, R. A. King, S. T. Brown, C. L. Janssen, E. T. Seidl, Y. Yamaguchi, W. D. Allen, Y. Xie, G. Vacek, T. P. Hamilton, C. B. Kellogg, R. B. Remington and H. F. Schaefer III (PSITECH Inc., Watkinsville, GA, 1999). 
[64] Stanton, J.F., Gauss, J., Lauderdale, W.J., Watts, J.D. and Bartlett, R.J. ACES II. The package also contains modified versions of the MOLECULE Gaussian integral program of J. Almlöf and P. R. Taylor, the ABACUS integral derivative program written by T. U. Helgaker, H. J. As. Jensen, P. Jorgensen, and P. R. Taylor, and the PROPS property evaluation integral code of P. R. Taylor.

[65] Klopper, W. (1997) J. Comp. Chem., 18, 20-27.

[66] DALTON, an ab initio electronic structure program, Release 1.0 (1997), written by Helgaker, T., Jensen, H.J.Aa., Jørgensen, P., Olsen, J., Ruud, K., Agren, H., Andersen, T., Bak, K.L., Bakken, V., Christiansen, O., Dahle, P., Dalskov, E.K., Enevoldsen, T., Fernandez, B., Heiberg, H., Hettema, H., Jonsson, D., Kirpekar, S., Kobayashi, R., Koch, H., Mikkelsen, K.V., Norman, P., Packer, M.J., Saue, T., Taylor, P.R. and Vantras, O.

[67] MOLFDIR, Aerts, P.J.C., Visser, O., Visscher, L., Merenga, H., de Jong, W.A. and Nieuwpoort, W.C., University of Groningen, The Netherlands.

[68] Visscher, L., Visser, O., Aerts, P.J.C., Merenga, H. and Nieuwpoort, W.C. (1994) Comp. Phys. Commun., 81, 120-144.

[69] Tennyson, J., Henderson, J.R. and Fulton, N.G. (1995) Comp. Phys. Comms., 86, 175-198.

[70] Polyansky, O.L., Jensen, P. and Tennyson, J. (1994) J. Chem. Phys., 101, 7651-7657.

[71] Tarczay, G., Császár, A.G., Polyansky, O.L. and Tennyson, J. (2001) J. Chem. Phys. to be submitted.

[72] Nielsen, I.M.B., Allen, W.D., Császár, A.G. and Schaefer III, H.F. (1997) J. Chem. Phys., 107, 1195-1211.

[73] Aarset, K., Császár, A.G., Sibert III, E.L., Allen, W.D., Schaefer III, H.F., Klopper, W., Noga, J. (2000) J. Chem. Phys., 112, 4053-4063.

[74] Valeev, E.F., Allen, W.D., Schaefer III, H.F., Császár, A.G. and East, A.L.L. (2001) J. Phys. Chem., submitted.

[75] Moazzen-Ahmadi, N. and Ozier, I. (1987) J. Mol. Spectrosc., 123, 26-36.

[76] Moazzen-Ahmadi, N. and Ozier, I., McRae, G. A. and Cohen, E. A. (1996) J. Mol. Spectrosc., 175, 54-61.

[77] Ozier, I. (1999) personal communication.

[78] Carter, S. and Handy, N.C. (1987) J. Chem. Phys., 87, 4294-4301.

[79] Jensen, P. (1989) J. Mol. Spectrosc., 133, 438-460.

[80] Polyansky, O.L., Jensen, P. and Tennyson, J. (1996) J. Chem. Phys., 105, 6490-6497.

[81] Lanquetin, R., Coudert, L.H. and Camy-Peyret, C. (1999) J. Mol. Spectrosc., 195, 54-67.

[82] Kain, J.S., Polyansky, O.L. and Tennyson, J. (2000) Chem. Phys. Lett., 317, 365-371.

[83] Valeev, E.F., Császár, A.G., Allen, W.D. and Schaefer III, H.F. (2001) J. Chem. Phys., accepted for publication.

[84] Evangelisti, S., Bendazzoli, G.L. and Gagliardi, L. (1994) Chem. Phys., 185, $47-56$. 
[85] Evangelisti, S., Bendazzoli, G.L., Ansaloni, R. and Rossi, E. (1995) Chem. Phys. Lett., 233, 353-358.

[86] Evangelisti, S., Bendazzoli, G.L., Ansaloni, R., Durí, F. and Rossi, E. (1996) Chem. Phys. Lett., 252, 437-446.

[87] Christiansen, O., Koch, H., Jørgensen, P. and Olsen, J. (1996) Chem. Phys. Lett., 256, 185-194.

[88] Olsen, J., Christiansen, O., Koch, H. and Jørgensen, P. (1996) J. Chem. Phys., 105, 5082-5090.

[89] Olsen, J., Jørgensen, P., Koch, H., Balková, A., and Bartlett, R.J. (1996) J. Chem. Phys., 104, 8007-8015.

[90] Sherrill, C.D., Van Huis, T.J., Yamaguchi, Y. and Schaefer III, H.F. (1997) J. Mol. Struct. (THEOCHEM), 400, 139-156.

[91] Ben-Amor, N., Evangelisti, S., Maynau, D. and Rossi, E. (1998) Chem. Phys. Lett., 288, 348-355.

[92] Rossi, E., Bendazzoli, G.L. and Evangelisti, S. (1998) J. Comp. Chem., 19, $658-672$.

[93] Sherrill, C.D., Leininger, M.L., Van Huis, T.J. and Schaefer III, H.F. (1998) J. Chem. Phys., 108, 1040-1049.

[94] Van Huis, T.J., Leininger, M.L., Sherrill, C.D. and Schaefer III, H.F. (1998) Coll. Czech. Chem. Comm., 63, 1107-1142.

[95] Leininger, M.L., Sherrill, C.D., Allen, W.D. and Schaefer III, H.F. (1998) J. Chem. Phys., 108, 6717-6721.

[96] Rossi, E., Bendazzoli, G.L., Evangelisti, S. and Maynau, D. (1999) Chem. Phys. Lett., 310, 530-536.

[97] Siegbahn, P.E.M., Blomberg, M.R.A. and Svensson, M. (1994) Chem. Phys. Lett., 223, 35-45.

[98] Brown, F.B. and Truhlar, D.G. (1985) Chem. Phys. Lett., 117, 307-313.

[99] Gordon, M.S. and Truhlar, D.G. (1986) J. Am. Chem. Soc., 108, 5412-5419.

[100] Leininger, M.L. and Császár, A.G. (2001) Mol. Phys. to be submitted.

[101] Feller, D. (1999) J. Chem. Phys., 111, 4373-4382.

[102] Feller, D. and Sordo, J.A. (2000) J. Chem. Phys., 113, 485-493.

[103] Feller, D. and Sordo, J.A. (2000) J. Chem. Phys., 112, 5604-5610.

[104] Chase Jr, M.W. (1998) J. Phys. Chem. Ref. Data, 9, 1.

[105] The following geometries $\left(\left[r_{\mathrm{OH}} / \AA, r_{\mathrm{OH}} / \AA, \theta_{\mathrm{HOH}} /\right.\right.$ degree $\left.]\right)$, in order of increasing energy, have been selected: [0.9583, 0.9583, 104.418], $[0.9583, \quad 0.9583,148.870], \quad[0.7784, \quad 1.1959, \quad 104.418], \quad$ and $[0.7784,0.7784,104.418]$.

[106] Martin, J.M.L. (1998) J. Chem. Phys., 108, 2791-2800.

[107] Polyansky, O.L., Jensen, P. and Tennyson, J. (1996) J. Mol. Spectrosc., 178, 184-188.

[108] Tennyson, J., Zobov, N.F., Williamson, R., Polyansky, O.L. and Bernath, P.F. (2001) J. Chem. Phys. Ref. Data, to be submitted.

[109] Polyansky, O.L., Zobov, N.F., Tennyson, J., Lotoski, J.A. and Bernath, P. (1997) J. Molec. Spectrosc., 184, 35-50. 
[110] Rothman, L.S., Gamache, R.R., Tipping, R.H., Rinsland, C.P., Smith, M.A.H., Benner, D.C., Malathy Devi, V., Flaud, J.-M., Camy-Peyret, C., Perrin, A., Goldman, A., Massie, S.T., Brown, L.R. and Toth, R.A. (1992) J. Quant. Spectrosc. Radiative Transf., 48, 469-507.

[111] Flaud, J.-M., Camy-Peyret, C. and Maillard, J.-P. (1976) Mol. Phys., 32, 499521.

[112] Polyansky, O.L., Busler, J.R., Guo, B., Zhang, K. and Bernath, P. (1996) J. Mol. Spectrosc., 176 305-315.

[113] Polyansky, O.L., Zobov, N.F., Viti, S. and Tennyson, J. (2000) J. Mol. Spectrosc., in press. 